\title{
Accuracy analysis of surface reconstruction from point clouds
}

\author{
A.N. Ruchay ${ }^{1,2}$, K.A. Dorofeev ${ }^{2}$, V.V. Kalschikov², A.V. Kober ${ }^{1}$ \\ ${ }^{1}$ Federal Research Centre of Biological Systems and Agro-technologies of the Russian Academy \\ of Sciences, 9 Yanvarya street 29, Orenburg, Russia, 460000 \\ ${ }^{2}$ Chelyabinsk State University, Bratiev Kashirinykh street 129, Chelyabinsk, Russia, 454001
}

\begin{abstract}
In this paper, we analyze the accuracy of surface reconstruction using point clouds. Various methods of surface reconstruction are tested and compared with respect to the reconstruction accuracy using real data. In order to improve the accuracy of 3D object reconstruction, an efficient method of surface reconstruction is designed. The presented results show an improvement in the accuracy of surface reconstruction using the proposed algorithm.
\end{abstract}

\section{Introduction}

Nowadays 3D high-quality model of real objects are needed in various areas such as medicine, agriculture, architecture, engineering, industrial metrology, cultural heritage preservation and restoration $[1,2,3]$. Accurate $3 \mathrm{D}$ object reconstruction is an important aspect for scene understanding, object retrieval, object tracking, object recognition, visualization and virtual maintenance $[4,5,6]$.

Noise and undefined holes on the surface can greatly affect to the accurate of 3D reconstruction $[7,8,9,10]$, therefore, noise-reduction and hole-filling enhancement algorithms should be served as a pre-processing step for $3 \mathrm{D}$ reconstruction $[11,12]$. To reduce impulsive noise and to fill small holes, filters are used [13, 14, 15, 16, 17].

In this paper, we are interested in the design of a surface reconstruction algorithm to improve the quality of 3D reconstruction $[18,19,20]$. Different evaluation criteria are used for surface reconstruction $[18,19,21,20]$. A common method of evaluation is to directly compare the geometry of the reconstruction output to the ground-truth surface. However, direct comparison with ground-truth data is insufficient when the reconstruction is performed under an error tolerance or it consists of several levels of details [20].

Our main goal is to evaluate surface reconstruction methods in terms of the reconstruction accuracy, which depends on the quality of the input mesh. We evaluate the performance of surface reconstruction methods with respect to the accuracy of $3 \mathrm{D}$ object reconstruction. Actually, we calculate the Hausdorf distance between the point cloud of the accurate laser model and point cloud of the restored mesh, the cloud to mesh distance between point cloud of accurate laser model and the restored mesh, and the mesh to mesh distance between the mesh of accurate laser model and the restored mesh. 
The performance of the proposed algorithm is compared in terms of the accuracy of 3D object reconstruction and speed with that of the following surface reconstruction algorithms: the marching cubes (MB) [22], the point set surface reconstruction for VTK (PSSRVTK) [23], the Poisson surface reconstruction algorithm in the VTK framework (PSRVTK) [24], the implicit hierarchical B-Splines surface reconstruction based on octree distance field (BSR) [25], the reconstruction of a triangle mesh from a set of oriented 3D points by solving a Poisson system (RTM) [26], the interactive surface reconstruction software with curve-driven controls (ISR) [27], the field-aligned online surface reconstruction (FAOSR) [28], the Ball-pivoting algorithm for surface reconstruction (BPSR) [29], the Poisson surface reconstruction (PSR) [30], the Power Crust algorithm (PC) [31].

The paper is organized as follows. Computer simulation results are provided in Section 2. Finally, Section 3 summarizes our conclusions.

\section{Experimental results}

In this section, we evaluate the performance of the tested surface reconstruction methods from point clouds in terms of the reconstruction accuracy.

Extensive experiments revealed that very good denoising results cannot be achieved using the following filters: MB [22], PSSRVTK [23], PSRVTK [24], BSR [25], RTM [26], ISR [27], FAOSR [28]. The main reason is incorrect mesh after surface reconstruction, therefore, we do not use these algorithms for our next experiments and comparisons.

We compare the following surface reconstruction algorithms: BPSR [29], PSR [30], PC [31]. The experiments are carried out on a PC with Intel(R) Core(TM) i7-4790 CPU @ $3.60 \mathrm{GHz}$ and 16 GB memory.

In our experiments we use the meshes of lion, apollo, and anatomy from the dataset [21].

We construct couples of meshes for each model using the following steps:

(i) Registration RGB and depth data.

(ii) Making point clouds.

(iii) Making input meshes from point clouds.

(iv) Calculating Hausdorf distance (HD) between point cloud of accurate laser model and point cloud of mesh, and cloud to mesh distance (CMD) between point cloud of accurate laser model and mesh, and mesh to mesh distance (MMD) between mesh of accurate laser model and mesh.

The calculated HD, CMD and MMD metrics for models of lion, apollo, and anatomy by PSR, BPSR, PC algorithms are shown in Tables 1 and 2. The BPSR algorithm yields the best result in terms HD, CMD and MMD among all surface reconstruction algorithms. The BPSR algorithm yields good results in terms of objective and subjective criteria.

As a result of our analysis, we found that the use of all three algorithms is possible only in the case when the input point cloud does not have areas of low point density. However, it is worth noting that the PC algorithm least distorts the shape, but the meshes resulting from this algorithm must be processed by mesh simplification algorithms. Also, based on the HD and MMD metrics, we can conclude that the BPSR algorithm is least changed by the original cloud.

\section{Conclusion}

In this paper, we compared various surface reconstruction algorithms in terms of the accuracy of 3D object reconstruction using real data from a RGB-D sensor. Experimental results have shown that the BPSR algorithm yields the best result in terms of the accuracy of 3D object reconstruction among all surface reconstruction algorithms. 
Table 1. Calculated HD, CMD and MMD metrics between accurate laser model of anatomy, apollo, lion and reconstructed model from accurate point cloud using BPSR, PSR, PC algorithms.

\begin{tabular}{lllll}
\hline Object & Algorithms & HD & CMD & MMD \\
\hline Anatomy & BPSR & $\mathbf{0}$ & $\mathbf{0}$ & $\mathbf{0}$ \\
& PSR & 0.07872 & 0.00002 & 0.00003 \\
\multirow{4}{*}{ Apollo } & PC & 0.08118 & 0.00022 & 0.00014 \\
& BPSR & $\mathbf{0}$ & $\mathbf{0}$ & $\mathbf{0}$ \\
& PSR & 0.09681 & 0.0006 & 0.00054 \\
Lion & PC & 0.06522 & 0.0003 & 0.00012 \\
& BPSR & $\mathbf{0}$ & $\mathbf{0}$ & $\mathbf{0}$ \\
& PSR & 0.06433 & 0.0003 & 0.00013 \\
& PC & 0.06757 & 0.00017 & 0.00013 \\
\hline
\end{tabular}

Table 2. Calculated HD, CMD and MMD metrics between accurate laser model of anatomy, apollo, lion and reconstructed model from real Kinect point cloud using BPSR, PSR, PC algorithms.

\begin{tabular}{lllll}
\hline Object & Algorithms & HD & CMD & MMD \\
\hline Anatomy & BPSR & 0.270656 & $\mathbf{0 . 0 0 0 3 4}$ & $\mathbf{0 . 0 0 0 1}$ \\
& PSR & 0.268457 & 0.00733 & 0.0032 \\
\multirow{4}{*}{ Apollo } & PC & 0.270657 & 0.003 & 0.0032 \\
& BPSR & 0.142087 & $\mathbf{0 . 0 0 0 1 2}$ & $\mathbf{0 . 0 0 0 1}$ \\
& PSR & 0.139367 & 0.00332 & 0.00386 \\
Lion & PC & 0.141812 & 0.002 & 0.00283 \\
& BPSR & 0.081381 & $\mathbf{0 . 0 0 0 3}$ & $\mathbf{0 . 0 0 0 1 3}$ \\
& PSR & 0.079486 & 0.00312 & 0.00314 \\
& PC & 0.081338 & 0.00296 & 0.0036 \\
\hline
\end{tabular}

\section{Acknowledgments}

This work was supported by the Russian Science Foundation, grant no. 17-76-20045.

\section{References}

[1] Ruchay, A. 3d object reconstruction using multiple kinect sensors and initial estimation of sensor parameters / A. Ruchay, K. Dorofeev, A. Kober // Proc. SPIE. - 2018. - Vol. 10752. P. 1075222.

[2] Ruchay, A. Fusion of information from multiple kinect sensors for 3d object reconstruction / A. Ruchay, K. Dorofeev, V. Kolpakov // Computer Optics. - 2018. - Vol. 42(5). - P. 898-903. DOI: 10.18287/2412-6179-2018-42-5-898-903.

[3] Ruchay, A.N. A depth camera-based system for automatic measurement of live cattle body parameters / A.N. Ruchay, K.A. Dorofeev, V.V. Kalschikov // IOP Conference Series: Earth and Environmental Science. - 2019. - Vol. 341. - P. 012148.

[4] Ruchay, A. Accuracy analysis of 3d object reconstruction using rgb-d sensor / A. Ruchay, K. Dorofeev, A. Kober // CEUR Workshop Proceedings. - 2018. - Vol. 2210. - P. 82-88.

[5] Ruchay, A. Accurate reconstruction of the $3 \mathrm{~d}$ indoor environment map with a rgb-d camera based on multiple icp / A. Ruchay, K. Dorofeev, A. Kober // CEUR Workshop Proceedings. - 2018. - Vol. 2210. - P. 300-308.

[6] Ruchay, A. Accurate 3d shape recovery of live cattle with three depth cameras / A.N. Ruchay, K.A. Dorofeev, V.V. Kalschikov // IOP Conference Series: Earth and Environmental Science. - 2019. - Vol. 341. - P. 012147. 
[7] Makovetskii, A. An efficient point-to-plane registration algorithm for affine transformations / A. Makovetskii, S. Voronin, V. Kober, D. Tihonkih // Applications of Digital Image Processing XL / International Society for Optics and Photonics. - 2017. - Vol. 10396. - P. 513-522.

[8] Makovetskii, A. Affine registration of point clouds based on point-to-plane approach / A. Makovetskii, S. Voronin, V. Kober, D. Tihonkih // Procedia Engineering. - 2017. - Vol. 201. - P. 322-330.

[9] Makovetskii, A. A non-iterative method for approximation of the exact solution to the point-toplane variational problem for orthogonal transformations / A. Makovetskii, S. Voronin, V. Kober, A. Voronin // Mathematical Methods in the Applied Sciences. - 2018. - Vol. 41(18). - P. 9218-9230.

[10] Ruchay, A. Accuracy analysis of 3d object reconstruction using point cloud filtering algorithms / A. Ruchay, K. Dorofeev, V. Kalschikov // CEUR Workshop Proceedings. - 2018. - Vol. 2391. - P. 169-174.

[11] Ruchay, A. Accuracy analysis of 3d object shape recovery using depth filtering algorithms / A. Ruchay, K. Dorofeev, A. Kober // Proc. SPIE. - 2018. - Vol. 10752. - P. 1075221.

[12] Ruchay, A. An efficient detection of local features in depth maps / A. Ruchay, K. Dorofeev, A. Kober // Proc. SPIE. - 2018. - Vol. 10752. - P. 1075223.

[13] Ruchay, A. Clustered impulse noise removal from color images with spatially connected rank filtering / A. Ruchay, V. Kober // Proc. SPIE. - 2016. - Vol. 9971. - P. 99712Y10.

[14] Ruchay, A. Removal of impulse noise clusters from color images with local order statistics / A. Ruchay, V. Kober // Proc. SPIE. - 2017. - Vol. 10396. - P. 1039626.

[15] Ruchay, A. Impulsive noise removal from color video with morphological filtering / A. Ruchay, V. Kober // Proc. SPIE. - 2017. - Vol. 10396. - P. 1039627.

[16] Ruchay, A. Impulsive noise removal from color images with morphological filtering / A. Ruchay, V. Kober // Analysis of Images, Social Networks and Texts - Cham: Springer International Publishing, 2018. - P. 280-291.

[17] Ruchay, A. Removal of impulsive noise from color images with cascade switching algorithm / A, Ruchay, A. Kober, V. Kolpakov, T. Makovetskaya // Proc. SPIE. - 2018. - Vol. 10752. - P. 1075224.

[18] Lim, S.P. Surface reconstruction techniques: A review / S.P. Lim, H. Haron // Artificial Intelligence Review. - 2012. - Vol. 42.

[19] Khatamian, A. Survey on 3d surface reconstruction / A. Khatamian, H. Arabnia // Journal of Information Processing Systems. - 2016. - Vol. 12. - P. 338-357.

[20] Berger, M. A survey of surface reconstruction from point clouds / M. Berger, A. Tagliasacchi, L.M. Seversky // Comput. Graph. Forum. - 2017. - Vol. 36(1). - P. 301-329.

[21] Lee, K. Realistic surface geometry reconstruction using a hand-held RGB-D camera / K. Lee, T.Q. Nguyen // Mach. Vis. Appl. - 2016. - Vol. 27(3). - P. 377-385.

[22] Lorensen, W.E. Marching cubes: A high resolution 3d surface construction algorithm / W.E. Lorensen, H.E. Cline // SIGGRAPH Comput. Graph. - 1987. - Vol. 21(4). - P. 163-169.

[23] Doria, D. Point set surface reconstruction for vtk// VTK Journal, 2011.

[24] Doria, D. Poisson surface reconstruction for vtk / D. Doria, A. Gelas // VTK Journal, 2010.

[25] Tang, Y. Multi-scale surface reconstruction based on a curvature-adaptive signed distance field /

Y. Tang, J. Feng // Computers \& Graphics. - 2018. - Vol. 70. - P. 28-38.

[26] Kazhdan, M. Screened poisson surface reconstruction / M. Kazhdan, H. Hoppe // ACM Trans. Graph. - 2013. - Vol. 32(3). - P. 29:1-29:13.

[27] Yin, K. Morfit: Interactive surface reconstruction from incomplete point clouds with curvedriven topology and geometry control / K. Yin, H. Huang, H. Zhang // ACM Trans. Graph. - 2014. Vol. 33(6). - P. 202:1-202:12.

[28] Schertler, N. Field-aligned online surface reconstruction / N. Schertler, M. Tarini, W. Jakob // ACM Trans. Graph. - 2017. - Vol. 36(4). - P. 77:1-77:13. 
[29] Bernardini, F. The ball-pivoting algorithm for surface reconstruction / F. Bernardini, J. Mittleman, H. Rushmeier // IEEE Transactions on Visualization and Computer Graphics. - 1999. Vol. 5(4). - P. 349-359.

[30] Kazhdan, M. Poisson surface reconstruction / M. Kazhdan, M. Bolitho, H. Hoppe // Proceedings of the Fourth Eurographics Symposium on Geometry Processing. - Aire-la-Ville, Switzerland, Switzerland : Eurographics Association, 2006. - P. 61-70.

[31] Amenta, N. The power crust / N. Amenta, S. Choi, R.K. Kolluri // Proceedings of the Sixth ACM Symposium on Solid Modeling and Applications. - New York, USA: ACM, 2001. - P. 249-266. 